\title{
Einfluss der intensiven Gruppenhaltung und Geschlecht auf Oral- Stereotypien und einige Verhaltensmerkmale beil Mastlämmern
}

\section{Summary}

Title of the paper: Effect of intensive group condition and sex on the oral stereotypes and some behaviour traits in fattening lambs

Oral stereotypes are a kind of the forms of behavioural abnormalities and many husbandry factors can cause the development of such behaviours in almost all domestic species. In this study, effects of housing based on group condition and also sex differences on the development of oral stereotypes were investigated in fattening lambs. Ten male and ten female Turkgeldi synthetic breed of lambs were used. Animals were assigned to sex groups at the beginning of the study and housed in pens that consisted of metal feed through and water buckets on a straw bedded floor during the experiment. Behavioural observations were taken by two observer and time sampling lechnique was used. Activities such as biting, licking and chewing of through, water bucket and also bars of pen were recorded as oral stereotypes. Generally, it was indicated that intensive group condition in fattening lambs causes the development of behavioural abnormalities related to nutritional origin. Activities towards the bedding maxerial, standing, lying and wool chewing behaviours were significantly affected by sex. The male lambs showed more activities in standing and wool licking and wool chewing behaviours than the females while the lying and activity towards the bedding material observed more frequently in the female group. This finding indicated that general activity was high in the male lambs. There was no significant sex effect on the total oral stereotypes. It was concluded that lack of structural materials have an important role in the development of oral stereotypes. In addition some stressful conditions that caused by group housing may also resulted in the development of such abnormalities especially for the male lambs.

Key Words: behaviour, stereotypes, abnormal behaviour, lamb, group housing, fattening

\section{Zusammenfassung}

Oral-Stereotypien sind eine artubergreifende Form von Verhaltensstorungen. In dieser Studie wurde der Frage nachgegangen, wie sich die Gruppenhaltung bei Mastlammem auf die Stereotypien auswirkt. Daneben wurde auch der Geschlechtseffekt untersucht. Als Material standen 10 männliche und 10 weibliche Lammer der synthetischen Rasse Türkgeldi zur Verfügung. Die Geschlechter wurden getrennt in nebeneinander eingerichteten Buchten, auf Stroheinstreu, gehalten. Die Beobachtungen erfolgten mit zwei Personen nach der Teil-Zeit-Methode. Es wurden die Verhaltensweisen aus dem Ernahrungsbereich und Ruhebereich erfasst. Als stereotype Maultatigkeiten wurden Futterkrippe-Lecken, Futterkrippe-Knabbern, Abtrenngitter-Lecken und AbtrenugitterKnabbern definiert. Die intensive Gruppenhaltung von Mastlammern fuhrte zu Verhaltensstorungen im Bereich des Fressverhaltens. Das Geschlecht hatte einen hoch signifikanten Einfluß auf die Merkmale Einstreu-Fressen, Stehen, Liegen und Wolleknabbern und -lecken (WKL). Die männlichen Lämmer zeigten eine hohere Hăufigkeit bei den Merkmalen Stehen und WKL, hingegen die weiblichen Lämmer bei den Merkmalen Einstreu-Fressen und Liegen. Die Ergebnisse deuteten auf eine höhere Aktivităt bei den männlichen Lämmem gegenüber den weiblichen Lämmern hin. Die Analyse der zusammengefassten stereotypen Maultatigkeiten ergab kejnen signifikanten Geschlechtseinfluß. Als mögliche Erkïrung des Vorkommens von stereotypen Maulaktivităten komm das Fehlen von strukturierter Nahrung in Frage. Des weiteren ist vermutlich der soziale Stress bei der Gruppenhaltung, der sich besonders auf die männlichen Lămmer auswirkt, eine Ursache der Stereotypien.

Schlisselworter: Verhalten, Stereotypie, Verhaltensstönung, Lamm, Gruppenhaltung, Mast

1. Einleitung

In der Türkei basiert die Schafhaltung bzw, -mast traditionell auf der Weidemast. Jedoch zwingen die steigende Bevölkerungszahl und die zurilckgehenden Weideflächen 
die Schafhalter zu einer Intensivierung ihrer Wirtschaftszweige. So hat in den letzten Yahren bei Lämmern die intensive Stallmast zugenommen. Insbesondere beim Schaf als Weidetier kann die Umwelteinschränkung in Form der Stallhaltung zu Verhaltensstörungen führen. Verhaltensstörungen werden in der Regel auf Mangelernährung zurückgeführt, was nicht immer zutrifft (SAMBRAUS und GOTTHARDT, 1985). Die Tiere haben z.B. neben Nahrungsbedarf, der umfangreich erforscht ist, auch einen Verhaitensbedarf (SMIDT et al., 1995).

Eine artübergreifende Verhaltensstörungsgruppe bilden die stereotypen Maultätigkeiten (BRUMMER, 1978; SAMBRAUS und GOTTHARDT, 1985; SAMBRAUS et al, 1984; COOPER et al., 1994; COOPER et al,, 1995; MORGAN et al., 1995; COOPER et al., 1996; REDPO et al., 1996). In dieser Studie wird der Frage nachgegangen, ob die intensive Gruppenhaltung bei Mastlämmern zu stereotypen Maultätigkeițen führt, Ferner wurde der Effekt des Geschlechts auf die stereotypen Oralaktivitäten sowie einige Verhaltensmerkmale untersucht.

2. Material und Methode

Tiere und Management

Die Untersuchung wurde an 10 männlichen und 10 weiblichen Türkgeldi Lämmern im Versuchsstall der Agrarwissenschaftlichen Fakultät zu Tekirdağ durchgeführt. Die Rasse Türkgeldi ist eine synthetische Schafrasse, die auf der Kreuzung des ostfriesischen Milchschafes und des Kivircik-Schafes (lokale Schafrasse aus der Nordwest Türkei) basiert. Die Tiere wurden getrennt nach Geschlechtern in nebeneinander liegenden Buchten auf Stroheinstreu gehalten. Es wurde nur am Beginn des Versuches eingestreut. Das durchschnittliche Gewicht bei der Aufstallung, die eine Woche vor Versuchsbeginn erfolgte, betrug bei den männlichen Lämmern $24,8 \pm 1,84 \mathrm{~kg}$ und bei den weiblichen Lämmern $23,4 \pm 2,53 \mathrm{~kg}$. Die Fütterung erfolgte zweimal am Tag um $9^{\frac{00}{0}}$ und $16^{00}$ Uhr. Dabei erhielten die Tiere Kraftfutter ad libitum in Metallkrippen. Wasser erhielten die Tiere ad libitum in Plastikbehältern. Zur individuellen Erkennung wurden die Lämmer mit Farbmarkierungen nummeriert.

Der Versuch erstreckte sich über sieben Wochen. Die Beobachtungen wurden einmal in der Woche, am Vormittag von $10^{00}$ bis $12^{\frac{30}{0}}$ Uhr und am Nachmitag von $15^{00}$ bis $16^{\frac{00}{2}}$, von zwei Personen nach der Zeit-Teil-Methode durchgeführt (BOGNER, 1984). Die Beobachter nahmen jeweils 15 Minuten vor Versuchsbeginn ihre Plätze ein, wobei die Tiere schon früher an diese Personen gewöhnt wurden. Es wurde in Intervallen von 5 Minuten das Verhalten des einzelnen Tieres notiert. Somit ergaben sich pro Lamm je Tag 42 Beobachtungspunkte.

Folgende Verhaltensmerkmale wurden erfasst:

Fressen (F)
Trinken (T)
Einstreu-Fressen (EF)
Wiederkauen (W)
Siehen (S)
Liegen (L)
Wolle-Knabbern oder -Lecken (WKL)
Sonstige Verhalten (SV)

:Futteraufnahme

: Wasseraufnahme

: Beschätigung mit dem Einstreu, uberwiegend Strohaufnahme

: Kaubewegungen in der Ruhelage (stehend oder liegend)

: Das Tier steht und kein weitercs Verhalten wird beobachtet

Das Tier liegt und kein weiteres Verhalten wird beobachtet

; Stereotypes Knabbem, beißen, Lecken oder Ziehen an der Wolle

: Sexualverhalten, Spielverhalien, Auseinandersetzungen, sich Kratzen oder Reiben am Abtrenngitter etc.

\section{Definition}


Die folgenden Stereotypien wurden nach den Maultatigkeiten und dem Material, an dem das Verhaiten ausgeubt wird, eingeteilt. Die Einteilung beruht auf der Annahme, dass diese Verhaltensstörungen auf unterschiedliche Ursachen zuruckzuführen sind (COOPER et al., 1996).

\footnotetext{
Stereotypie

Futterkrippe-Lecken (FL)

Futterkrippe-Knabbern (FK)

Abtrenngitter-Lecken ( $\mathrm{AL}$ )

: Stereotypes Lecken an der Futterkrippe mit Lippenbewegungen und der Zunge

: Stereotypes Knabbern und Beißen an der Futterkrippe

: Stereotypes Lecken am Abtrenngitter mit Lippenbewegungen und der Zunge

Abtrenngitter-Knabbem (AK)

: Stereotypes Knabbem und Beißen am Abtrenngitter
}

Die oben genannten Stereotypien wurden auch zusammenfassend (Stereotype OralAktivitäten (SOA)) analysiert.

\section{Statistische Auswertung}

Unter der Annahme, dass die wiederholten Beobachtungen an einem Lamm nicht korreliert sind, wurde zur statistischen Auswertung das folgende Schwellenwertmodell angewandt:

$$
\pi_{i j k}=\Phi\left(G_{i}+T_{j}+P_{k}\right)
$$

Dabei stellt $\pi_{\mathrm{ijk}}$ den erwarteten Anteil der Tiere dar, die das entsprechende Verhalten ausüben. Die kumulative Wahrscheinlichkeitsfunktion fur die Standardnormalverteilung wird durch $\Phi$ gekennzeichnet, $G_{i}$ als fixer Effekt des Geschlechts, $T_{j}$ als fixer Effekt des Beobachtungstages und $\mathbf{P}_{\mathrm{k}}$ als fixer Effekt der Tageszeit.

Die statistische Analyse wurde mit der SAS-Prozedur GENMOD durchgeführt (SAS, 1992). Die einzelnen Einflussgrößen wurden mit Hilfe der geschătzten Regressionskoeffizienten (b), deren Standardfehler und der „odds ratios" $(\psi)$ beurteilt. „Odds ratio" wird dabei als das Verhăltnis der Wahrscheinlichkeit, dass ein Verhalten ausgeubbt wird, zur Wahrscheinlichkeit, dass das entsprechende Verhalten nicht ausgeübt wird. Sie werden aus den geschătzten Regressionskoeffizienten der jeweiligen Effekte berechnet $\left(\psi=e^{b}\right)$.

\section{Ergebnisse und Diskussion}

\section{Phänotypische Häufigkeiten}

In Tabelle 1 sind die phänotypischen Hăufigkeiten der beobachteten Verhaltensmerkmale wiedergegeben. Die Rangierung nach der Häufigkeit ist für die Verhaltensmerkmale Liegen (L), Stehen (S) und Fressen (F) bei den Geschlechtern identisch. Sogar die kumulativen Häufigkeiten dieser Verhaltensmerkmale, mit 84,7\% für die weiblichen Lämmer und mit 85,5\% für die männlichen Lämmer, sind sehr ähnlich. Obwohl die kumulativen Häufigkeiten dieser Merkmale ähnlich sind, ist die Differenz bei den Merkmalen Stehen (S) und Liegen (L) sehr hoch.

Die Wasseraufnahme ist von mehreren Umweltfaktoren abhängig (YURTMAN et al., 1996). Da die Umwelteinflüsse auf die Geschlechter gleich sind und entsprechend der ähnlichen Häufigkeiten des Fressens auch ähnliche Häufigkeiten zwischen den Geschlechtern beim Trinkverhalten erwartet werden, stimmen die Beobachtungshäufigkeiten des Trinkens für die Geschlechter gut mit den Erwartungen überein. 
Da die meisten Stereotypien auf reizarme Umwelt und das Fehlen von strukturierter Nahrung zurtkckgefuhrt werden (SAMBRAUS et aI., 1984; SAMBRAUS und GOTTHARDT, 1985; DÜRING et al., 1989), sind Wechselwirkungen zwischen den Verhaitensmerkmalen Einstreu-Fressen (EF), Wolle-Knabbern oder-Lecken (WKL) und der stereotypen Oral-Aktivitäten (SOA) zu erwarten. Ein Vergleich der kumulativen Häufigkeiten der Merkmale EF, WKL und SOA bei den Geschlechtern zeigt hingegen eine hohe Differenz zwischen den Geschlechtern (weibliche Tiere 9,09\%, männliche Tiere $6,87 \%$ ). Verursacht wird diese Differenz möglicherweise dadurch, dass die mănnlichen Tiere ihren Stress zusătzlich uber Sozialverhalten abreagieren.

Tabeile I

Phänotypische Haufigkeiten der beobachteten Verhaltensmerkmale, getrennt nach Geschlechtern (Frequencies of behavioural observations in the groups)

\begin{tabular}{lcc}
\hline Verhaltensmerkmale, \% & Weibliche Lämmer & Männiiche Lämmer \\
\hline Fressen & 10,29 & 10,55 \\
Trinken & 1,06 & 1,16 \\
Einstreu-Fressen & 5,59 & 1,40 \\
Wiederkauen & 3,20 & 2,92 \\
Stehen & 18,30 & 30,98 \\
Liegen & 56,07 & 44,00 \\
Wolle-Knabbem oder -Lecken & 0,95 & 3,37 \\
Stereotype Oral-Akkivitaien & 2,55 & 2,10 \\
Sonskiges Verhaiten & 1,98 & 3,51 \\
\hline
\end{tabular}

Die phänotypischen Häufigkeiten der einzelnen Stereotypien, die in der Tabelle 2 zusammengefasst sind, lassen, hinsichtlich der Maulaktivitäten oder des Materials, an dem die entsprechende Stereotypie ausgeübt wird, keinen Trend erkennen. Während die weiblichen Tiere das Lecken $(1,36 \%)$ häufiger zeigen als das Knabbern $(1,19 \%)$, zeigen die männlichen Tiere das Knabbern häufiger $(1,22 \%)$ als das Lecken $(0,88 \%)$. Der gleiche Trend ist auch für das Material zu beobachten. Während die weiblichen Tiere die stereotypen Maulaktivitäten häufiger an der Futterkrippe ausüben, richten sich die Aktivitäten der männlichen Tiere häufiger gegen das Abtrenngitter.

Tabelle 2

Phănotypische Hăufigkeiten der beobachteten Stereotypien, getrennt nach Geschlechtern (Frequencies of stereotypic behaviours in the groups)

\begin{tabular}{lcc}
\hline Sterectypie & Weibliche Lämmer & Mănnliche Lämmer \\
\hline Futterkrippe-Lecken & 1,12 & 0,71 \\
Futterkrippe-Knabbern & 0,82 & 0,27 \\
Abtrenngitter-Lecken & 0,24 & 0,17 \\
Abrenngitter-Knabbern & 0,37 & 0,95 \\
\hline
\end{tabular}

Verlauf der durchschnittlichen Häufigkeiten während des Untersuchungszeitraumes und im Tagesablauf

Das Merkmal Fressen zeigt in den beiden Geschlechtern, während der gesamten Untersuchungsperiode, nahezu einen konstanten Verlauf (Abb. 1). Die anfänglich relativ hohe Einstreuaufnahme (EF), die bei den weiblichen Tieren zu beobachten ist, sinkt in der vierten Woche und bleibt danach bis zum Versuchsende konstant. Da eine Beziehung zwischen Wiederkauen und Aufnahme von strukturierter Nahrung zu erwarten ist, stimmt der ähnliche Verlauf der Merkmale W und EF bei den weiblichen 
Tieren gut mit den Erwartungen überein. Im Gegensatz dazu, ist bei den männlichen Lämmern die Erhöhung des Merkmales W in der dritten und vierten Woche erklärungsbedürftig. Deutliche Unterschiede zwischen den Geschlechtern werden bei den Merkmalen Stehen und Liegen beobachtet. Während bei den weiblichen Lämmern die Schwankungen der genannten Merkmale ziemlich gering sind, wird bei den männlichen Lämmern ein zunehmender Trend des Stehens und ein sinkender Trend des Liegens beobachtet. Diese gegensätzlichen Trends der Merkmale S und L deuten bei den männlichen Tieren auf eine Aktivitätszunahme hin. Beachtenswert ist der Trend des Merkmales Wolle-Knabbern oder -Lecken, das zwar in beiden Geschlechtern auf unterschiedlichem Niveau, aber doch tendenziell ähnlich verläuft. Dabei wird von den Stereotypien auf WKL übergegangen. WKL wird meistens auf Futtermangel zurückgeführt (SAMBRAUS, 1985). Die vorliegende Untersuchung allerdings gestattet keine klare Aussage furr den ansteigenden Trend der WKLHăufigkeiten.
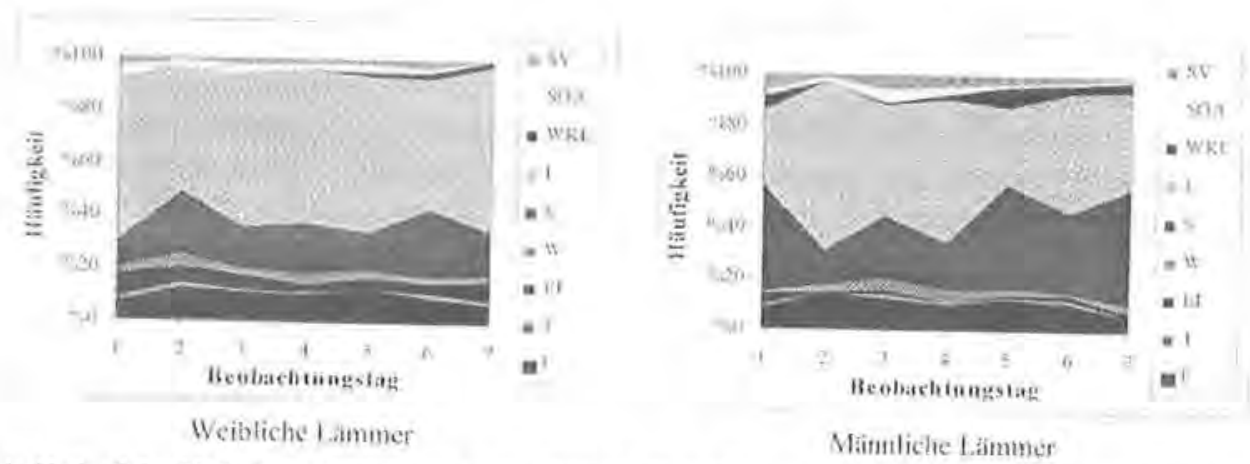
Abb, 1: Verlauf der Verhaltenshaufigkeiten fiber sieben Wochen (Changes in frequencies of behaviours during
seven weeks)

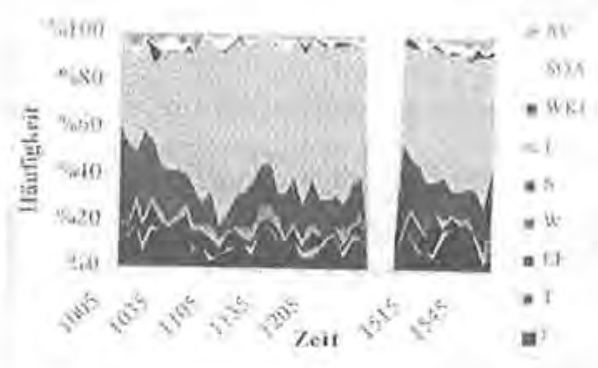

Weibliche Lảmmer

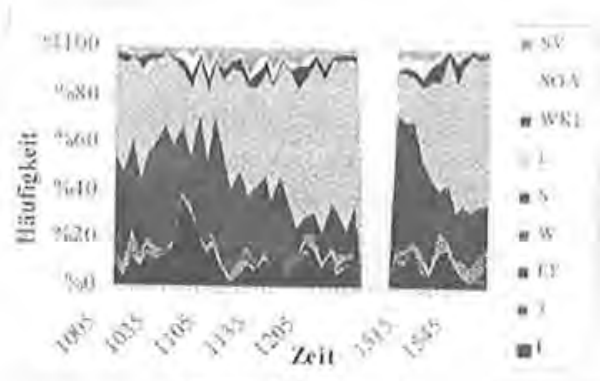

Männliche Lämmer

Abb. 2: Tagesverlauf der Verhaltenshafufigkeiten (Daily changes in the frequencies of behaviours)

In Abbildung 2 werden die Tagesabläufe der Verhaltensmerkmale getrennt für jedes Geschlecht wiedergegeben. Bei den weiblichen Tieren kann über den gesamten Beobachtungszeitraum kein eindeutiger Trend für das Fressen erkannt werden, wohingegen bei đen männilichen Tieren eine Hauptfresszeit zwischen $10^{30}$ und $11^{00}$ Uhr beobachtet wurde. Außer dieser Hauptfresszeit verlaufen die Häufigkeiten für das Fressen bei den 
männlichen Lämmern im niedrigeren Bereich als bei den weiblichen Lämmern. Die weiblichen Lämmer zeigen einen leichten Anstieg gegen die Mittagszeit bei dem Merkmal Trinken. Die männlichen Tiere trinken dagegen nach der Hauptfresszeit häufiger. Zwischen $10^{30}$ und $11^{00}$ Uhr, während der Hauptfresszeit der männlichen Tiere, ist ein Anstieg des Merkmales EF für die weiblichen Lämmer zu beobachten. Dennoch erreicht, bei den weiblichen Lämmern, die Summe der beiden Merkmale (F und EF) nicht die Hăufigkeit des Fressens der männlichen Tiere. Im weiteren wird bei den weiblichen Lämmern das Merkmal EF nachmittags häufiger beobachtet. Die männlichen Lämmer beschäftigen sich selten mit dem Einstreu, wobei kein eindeutiger Tagestrend zu erkennen ist.

Die höheren Häufigkeiten des Stehens zu Beginn der Beobachtungsperioden können nicht als Beobachterstorung interpretiert werden. Da die Futterung um $9^{00}$ Uhr erfolgte, erstreckt sich vermutlich die Futterungsunruhe bis zum Beobachtungsanfang. Im Gegensatz dazu muss die Nachmittagsbeobachtung des Merkmals Stehen anders interpretiert werden. Die Verläufe der Merkmale S und L bei den mănnlichen Tieren deuten auf eine Ruhezeit um die Mittagszeit. Bei den weiblichen Tieren hingegen zeigte sich keine Ruhezeit.

Das WKL wurde bei den mănnlichen Tieren durchgehend beobachtet, wobei bei der Nachmittagsbeobachtung ein leichter Anstieg zu sehen war. Die weiblichen Lämmer dagegen beschäftigten sich viel seltener mit der Wolle ihrer Buchtengefährten. Der Tagesverlauf der stereotypen Oral-Aktivitäten lässt keinen Trend erkennen. Sie verlaufen bei den weiblichen Tieren relativ konstant. Bei den männlichen Lämmern haben die SOA ein niedrigeres Niveau als bei den weiblichen Lämmern, wobei in der ersten halben Stunde der Nachmittagsbeobachtungen ein stärkerer Anstieg zu beobachten war.

Bezogen auf das sonstige Verhalten haben die weiblichen Lämmer eine geringere Häufigkeit als die männlichen Lämmer gezeigt, wobei wieđ̄erum bei den männlichen Lämmern ein Anstieg nach der Hauptfresszeit $\left(10^{30}\right.$ bis $\left.11^{\frac{00}{}}\right)$ beobachtet wurde.

\section{Analyse der Einflussgrößen}

In den. Tabellen 3 und 4 sind die Ergebnisse für die im Modell verwendeten Einflussfaktoren dargestellt. Während das Geschlecht keinen signifikanten Einfluss auf das Merkmal F ausübr, sind die Unterschiede der Verhaltenshäufigkeiten bezogen auf die Beobachtungstage und die Tageszeiten signifikant verschieden. Auf das Merkmal Trinken hatte nur die Tageszeit einen signifikanten Einfluss. Die Einflussfaktoren Geschlecht und Beobachtungstag sind im Gegensatz zur Tageszeit für das EF hoch signifikant. Beim Wiederkauen wurde kein signifikanter Geschlechtseffekt festgestelit, jedoch waren die Beobachtungstag- und die Tageszeiteffekte signifikant verschieden.

Das Geschlecht und der Beobachtungstag erwiesen sich als statistisch gesicherte Einflussfaktoren auf die Merkmale S, L und WKL. Im Gegenteil dazu hatte die Tageszeit keinen signifikanten Einfluss auf die genannten Merkmaie. Das Merkmal SOA wird nur vom Beobachtungstag signifikant beeinflusst.

Wie aus der Tabelle $4 \mathrm{zu}$ erkennen ist, wurden signifikante Geschlechtseffekte für die Merkmale FK und AK gefunden. Während die Merkmale FL und FK signifikante Unterschiede hinsichtlich der Häufigkeiten der Beobachtungstage zeigten, wurden bei 
Tabelle 3

Chi ${ }^{2}$-Werte $\left(\chi^{2}\right)$ und signifikanz Angaben (p) der Einflußgroßen auf die beobachteten Verhaltensmerkmalen (Chi-Square $\left(\chi^{2}\right)$ values of the main factors and their significance levels $(\mathrm{p})$ in the observed behaviours)

\begin{tabular}{|c|c|c|c|c|c|c|}
\hline \multirow[b]{2}{*}{ Verhaltensmerkmal } & \multicolumn{2}{|c|}{ Geschlecht } & \multicolumn{2}{|c|}{ Tag } & \multicolumn{2}{|c|}{ Tageszeit } \\
\hline & $x^{2}$ & $p$ & $x^{2}$ & $p$ & $x^{2}$ & $\mathrm{p}$ \\
\hline Fressen & 0,07 & n.s. & 35,84 & 600 & 9,13 & $\omega$ \\
\hline Trinken & 0,12 & n.s. & 5,15 & n.s. & 7,83 & $n$ \\
\hline Einstreu-Fressen & 84,08 & $\Leftrightarrow \phi$ & 31,57 & Des & 5,21 & * \\
\hline Wiederkauen & 0,59 & n.s. & 18,18 & i* & 7,23 & $\cdots$ \\
\hline Stehen & 124,16 & 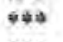 & 65,93 & * in & 1,94 & n.s. \\
\hline Liegen & 86,18 & ten & 49,30 & 948 & 1,18 & n.s. \\
\hline Wolle-Knabbern oder-Lecken & 42,89 & $\leftrightarrow 4$ & 92,43 & ats & 2,47 & n.s. \\
\hline Stereorype Oral-Aktivitäten & 0,83 & n.s. & 35,12 & $+\infty \%$ & 0,00 & n.s. \\
\hline
\end{tabular}

n.s.: nicht signifiksnt, " $p<0,05,{ }^{* 4} p<0,01,{ }^{* 04} p<0,001$

Tabelle 4

Chi $i^{2}$-Werte $\left(\chi^{2}\right)$ und Signifikanzangaben (p) der Einflussgroßen auf die beobachteten Stereotypien (Chi-Square $\left(\chi^{2}\right)$ vaiues of the main factors and their significance levels $(\mathrm{p})$ in the observed stereotypes)

\begin{tabular}{lcccccc}
\hline \multirow{2}{*}{ Siereotypie } & \multicolumn{3}{c}{ Geschlecht } & \multicolumn{2}{c}{ Tag } & \multicolumn{2}{c}{ Tageszeit } \\
& $\chi^{2}$ & $\mathrm{p}$ & $\chi^{2}$ & $\mathrm{p}$ & $\chi^{2}$ & $\mathrm{p}$ \\
\hline Futterkrippe-Lecken & 2,92 & n.s. & 44,86 & & 2,46 & n.s. \\
Futterikrippe-Knabbem & 8,27 & -6 & 14,63 & & 0,00 & n.s. \\
Abtrenngitter-Lecken & 0,30 & n.s. & 4,75 & n.s. & 0,86 & n.s. \\
Abtrenngitter-Knabbem & 7,82 & n. & 7,27 & n.s. & 0,05 & n.s. \\
\hline
\end{tabular}

n.s.: nicht signifikant, ${ }^{\circ} p<0,05,{ }^{\circ 0} p^{\circ}<0,01,{ }^{* 06} p<0,001$

Tabelle 5

Regressionskoeffizienten (b), deren Standardfehler (SE) und odds ratios $(\psi$ ) der Einflussgroßen und Verhaltensmerikmale (Regression (b) coefficients, standard errors (SE) and their odd ratios $(\psi)$ of the main factors and behavioural classes)

\begin{tabular}{|c|c|c|c|c|c|c|c|c|c|c|}
\hline \multirow[b]{3}{*}{ Merkma! } & \multicolumn{5}{|c|}{ Geschlecht } & \multicolumn{5}{|c|}{ Tageszeit } \\
\hline & \multicolumn{3}{|c|}{ Weiblich } & \multicolumn{2}{|c|}{ Männlich } & \multicolumn{3}{|c|}{ Vormittag } & \multicolumn{2}{|c|}{ Nachmittag } \\
\hline & b & SE & $\psi$ & b & $\psi$ & b & SE & $\psi$ & b & $\psi$ \\
\hline$F$ & $-0,01$ & 0,04 & 0,99 & 0,00 & 1,00 & 0,15 & 0,05 & 1,16 & 0,00 & 1,00 \\
\hline$T$ & $-0,03$ & 0,10 & 0,97 & 0,00 & 1,00 & 0,33 & 0,12 & 1,39 & 0,00 & 1,00 \\
\hline $\mathrm{EF}$ & 0,63 & 0,07 & 1,88 & 0,00 & 1,00 & $-0,16$ & 0,07 & 0,85 & 0,00 & 1,00 \\
\hline W & 0,05 & 0,06 & 1,05 & 0,00 & 1,00 & $-0,19$ & 0,06 & 0,83 & 0,00 & 1,00 \\
\hline S & $-0,40$ & 0,03 & 0,67 & 0,00 & 1,00 & $-0,06$ & 0,04 & 0,94 & 0,00 & 1,00 \\
\hline $\mathrm{L}$ & 0,31 & 0,03 & 1,36 & 0,00 & 1,00 & $-0,04$ & 0,03 & 0,96 & 0,00 & 1,00 \\
\hline WKL & $-0,54$ & 0,08 & 0,58 & 0,00 & 1,00 & $-0,13$ & 0,08 & 0,88 & 0,00 & 1,00 \\
\hline SOA & 0,06 & 0,07 & 1,06 & 0,00 & 1,00 & $-0,01$ & 0,07 & 0,99 & 0,00 & 1,00 \\
\hline
\end{tabular}

keiner Stereotypie signifikante Tageszeiteffekte beobachtet.

Ein Vergleich der Tabelle 1 mit den Tabellen 5 und 6 zeigt, dass die Regressionskoeffizienten und „odds ratios", die in der Tabelle 1 angegebenen phänotypischen Häufigkeiten für die Geschlechter widerspiegeln und die phänotypischen Beobachtungen bestätigen. Das Verhalten Fressen wird am Vormittag 16\% $\quad(\psi=1,16)$ häufiger ausgeubt als am Nachmittag. Noch höher ist die Hăufigkeit für das Trinken am Vormittag $(\psi=1,39)$. Im Gegensatz dazu zeigt das Merkmal EF am Nachmittag eine höhere Frequenz als am Vormittag $(\psi=0,85)$. Es wird vermutet, dass die Strohauf- 
nahme Durst nicht so hervorruft, wie Kraftfutter. Im weiteren ist zu bedenken, dass Durst von vielen unterschiedlichen Umweltfaktoren und deren Wechselwirkungen beeinflusst wird und das die Beobachtungen nicht die tatsächliche Wasseraufnahme erfassen.

Wie schon aus der Tabelle 1 zu ersehen ist, wird das Einstreu-Fressen bei den weiblichen Tieren häufiger beobachtet. Dabei ist für die weiblichen Lämmer der geschätzte „odds ratio"- Wert 1,88 und damit höher als bei männlichen Lämmern. Obwohl die weiblichen Lämmer mehr Stroh aufnehmen als die männlichen Lämmer, ist der Unterschied der Geschlechter beim Wiederkauen geringer als erwartet. Aufgrund der Beobachtungen kann angenommen werden, dass die Wolle auch eine stimulierende Wirkung auf das Wiederkauen hat, da bei den männlichen Tieren WKL häufiger beobachtet wird $(\psi=0,58)$. Allerdings muss beachtet werden, dass die EF und WKL Beachtungen nicht auf die absolute Aufnahme von Stroh bzw. Wolle hinweisen. Es wird jedoch eine hohe Korrelation zwischen den Beobachtungshäufigkeiten und der Stroh- bzw. Wolleaufnahme erwartet.

Tabelle 6

Regresionskoeffizienten (b), deren Standardfehler (SE) und odds ratios $(\psi$ ) der Einflußgroßen und Stereotypien (Regression coefficients (b), standard errors (SE) and their odd ratios $(\psi)$ of the main factors and stereotypies)

\begin{tabular}{lcccccccccc}
\hline & \multicolumn{3}{c}{$\begin{array}{c}\text { Weiblich } \\
\text { Merkmal }\end{array}$} & b & SE & $\psi$ & \multicolumn{2}{c}{ Männlich } & \multicolumn{3}{c}{ Vormittag } & b & $\psi$ & b & SE & $\psi$ & b & $\psi$ \\
\hline FL & 0,18 & 0,11 & 1,20 & 0,00 & 1,00 & $-0,18$ & 0,11 & 0,84 & 0,00 & 1,00 \\
FK & 0,38 & 0,14 & 1,46 & 0,00 & 1,00 & 0,00 & 0,14 & 1,00 & 0,00 & 1,00 \\
AL & 0,10 & 0,18 & 1,11 & 0,00 & 1,00 & 0,21 & 0,23 & 1,23 & 0,00 & 1,00 \\
AK & $-0,34$ & 0,12 & 0,71 & 0,00 & 1,00 & $-0,03$ & 0,12 & 0,97 & 0,00 & 1,00 \\
\hline
\end{tabular}

Die höhere Häufigkeit des Merkmales S und die niedrigere Häufigkeit des Merkmales L der männlichen Tiere gegenüber der weiblichen Tiere deuten auf eine höhere Aktivität bei den männlichen Lämmern hin, was mit der höheren Häufigkeit des sonstigen Verhaltens (Spiel- und Sexualverhalten, Auseinandersetzungen etc.) unterstützt wird (Tab. 1). Der soziale Kontakt mit dem Buchtengefährten ist bei den männlichen Tieren höher. Dieser enge Kontakt mit den Buchtengefährten hat zu einer häufigeren WKL bei den männlichen Tieren gegenüber der häufigeren EF bei den weiblichen Lämmern gefuhrt. Ein höheres EF der weiblichen Tiere wird bei den männlichen Tieren durch das WKL kompensiert. Die um die 6\%'ig geringer beobachteten stereotypen Maultätigkeiten der männlichen Tiere, die zwar nicht signifikant gesichert werden können, wäre auch als Hinweis auf eine höhere Aktivităt der månnlichen Tiere zu werten. Die signifikanten Unterschiede der Geschlechter, hinsichtlich der Merkmale FK und AK, scheinen diese Interpretation zu bestätigen (Tab. 4). So verrichten die weiblichen Lämmer ihr stereotypes Knabbern häufiger an der Futterkrippe, die männlichen Lämmer hingegen am Abtrenngitter (Tab. 6), d.h. dass die männlichen Tiere die Buchtenfläche besser ausnutzen als die weiblichen Tiere. Daraus kann wiederum auf eine höhere Aktivität der männlichen Tiere geschlossen werden. Ansonsten lassen die statistischen Analysen für die einzelnen Stereotypien keine Aussage hinsichtlich ihrer unterschiedlichen auslosenden Ursachen, die von COOPER et al. (1996) behauptet werden, zu. 
4.

Schlussfolgerungen

Die Ergebnisse zeigen, dass die intensive Gruppenhaltung bei Mastlämmern zu Verhaltensstörungen im Bereich des Fressverhaltens führen kann. Eine mögliche Erklärung ist ein Fehlen von strukturierter Nahrung, die durch Einstreuaufnahme zum Teil aufgehoben wird. Da mit dem Fortschreiten des Versuchs die Einstreu stärker verschmutzt wurde führte dieses zu einer Reduzierung der Aufnahme vom Einstreu.

In der vorliegenden Studie zeigten die stereotypen Maultätigkeiten im Vergleich mit einem Versuch, in dem Mastlämmer in Individualboxen gehalten wurden (YURTMAN et al., 2000), ein erhöhtes Auftreten. Als mögliche Ursache kann neben anderen Umweltreduzierungen auch sozialer Stress angesehen werden.

Im allgemeinen wurde eine höhere Aktivität bei den männlichen Lämmern beobachtet. Diese höhere Aktivität zeigte sich besonders im Bereich sozialer Auseinandersetzungen und Sexualverhalten, was wahrscheinlich auch zu einem häufigeren Wolleknabbern bei den männlichen Lämmern im Vergleich zu den weiblichen Lämmern geführt hat. Die aufgenommene Wolle bildet im Pansen Haarknäuel (Bezoar), die den Darm verstopfen und zum Eingehen des Tieres führen können. Außerdem erfordert eine höhere Aktivität einen höheren Energieaufwand (RAMONET et al., 2000). Eine artgerechtere Haltung, z.B. bei der intensiven Mast neben Krafffutter eine Heuzufütterung, würde die Zeit für die Futteraufnahme verlängern, was wiederum, besonders bei den männlichen Tieren, die Aktivität mildern würde.

BOGNER, $\mathrm{H}$ :

\section{Literatur}

Verhaltensbeobachtungen, Versuchsanlage und-auswertungen. In: H. BOGNER und A. GRAUVOGL, Verhalten landwirtschaftlicher Nutztiere, Verlag Eugen Ulmer, Stuttgart (1984), 61-74

BRUMMER, H.: Verhaltenstorungen. In: H.H. SAMBRAUS, Nutztierethologie, Verlag Paul Parey, Berlin-Hamburg,
(1978) $281-292$

COOPER, J.J.; EMMANS, G.C.; FRIGGENS, N.C.:

Effect of diet on behaviour of individually penned lambs. Animal Science 58 (1994), 441

COOPER, J.J.; McCULLAM, J.; SHANKS, M.:

The effect of fibrous diet on abnormal oral behaviour in stall howed lambs. British Society of Animal Science Winter Meeting, Summaries (1995) Paper 207

COOPER, J.; HASKELL, M.; LEWIS, R.M.:

The development of stereotypies in experimental sheep. Animal Science 62 (1996), 674

DÜING, F.; WAGNER, 1.; ERNST, E.; SCHLICHTING, M.C.; KALLWEIT, E.: Auswirkungen der Gruppenhaltung mit und ohne Einstreu auf Verhalten, Konstitution und Produktionsleistungen bei Mastlämmern. Zuchtungskunde 61 (1989), 141-ISI

MORGAN, C.A.; LAWRENCE, A.B.; HUNTER, F.A.; OLDHAM, J.D.;

Effect of meal frequency and rate of nutrient supply on the post-feeding behaviour of the pig. Animal Science 61 (1995), 565-573

RAMONET, Y.; BOLDUC, J.; BERGERON, R.; ROBERT, S.; MEUNIER-SALOÜN, M.C.: Feeding motivation in pregnant sows: effects of fibrous diet in an operant conditioning procedure. Appl. Anim. Behav. 66 (2000), 21-29

REDPO, 1.; EMANUELSON, M.; LUNDBERG, K.; OREDSON, N.: Feeding level and oral stereotypes in dairy cows. Animal Science 62 (1996), 199-206

SAMBRAUS, H.H.; KIRCHNER, M.; GRAF, B.:

Verhaliensstörungen bei intensiv gehaltenen Mastbullen. Dtsch. Tierärztl. Wschr. 91 (1984), 56-60

SAMBRAUS, H.H.; GOTTHARDT, A.:

Präputiumsaugen und Zungenspielen bei intensiv gehaltenen Mastbullen. Dtsch. Tierärztl. Wschr. 92 (1985), $465-468$ 
SAMBRAUS, H.H.:

Mouth-Based anomalous syndromes. In: A.F. FRASER, Ethology of farm animals. Elsevier, Oxford. Amsterdam, New York, Tokyo, (1985), 391-422

SAS INSTITUTE INC.:

User's Guide. Vers, 6.07, Cary, NC, USA (1992)

SMIDT, D.; SCHLICHTING, M.C.; LADEWIG, J.; STEINHARDT, M.:

Ethologische und verhaltensphysiologische Forschung fur tiergerechte Nutztierhaltung. Arch. Tierz. Dummerstorf 38 (1995), 7-19

YURTMAN, LY.; BAȘPINAR, E.; POLATSÜ, Ş.; OZKAN, M.M.; ÓZDÜVEN, M. L.:

The relationship of water intake and some environmental factors in fattening lambs. Besi kuzularinda bazı çevre faktörleri ile su tulketimi arasındaki ilişkiler. T.O.T. Ziraat Fakıltesi Yayınları Genel Yayın No: 253, Araștırma No: 98, Tekirdağ (1996)

YURTMAN, I.Y.; SAVAȘ, T; KARAAĞAC, F.; COȘKUNTUNA, L.:

Effects of daily protein intake levels in energy restricted lambs on the oral stereotypic behaviours. (2000) (in Vorbereitung)

Eingegangen: $31.05,2000$

Akzeptiert: 30.01 .2001

Anschrift der Verfasser

Dr. TÜRKER SAVAS, Doz. Dr. L. YAMAN YURTMAN, Tierarzt FEVZI KARAAĞAC.

Dr. ERTAN KÖYÜ

T.Ü.T. Ziraat Fakultesi

Zootekni Bolamu

59030 Tekirdag/TURKE! 